\title{
Discriminating Abiotic and Biotic Chemistry on Active Ocean Worlds
}

\author{
FABIAN KLENNER $^{1}$, FRANK POSTBERG ${ }^{1}$, JON $^{\prime}$ \\ HILLIER $^{1}$, NOZAIR KHAWAJA ${ }^{1}$, MARIE \\ DANNENMANN ${ }^{1}$, MORGAN L. CABLE $^{2}$, BERND \\ $\mathrm{ABEL}^{3}$
}

${ }^{1}$ Freie Universität Berlin, Germany (f.klenner@fu-berlin.de)

${ }^{2}$ NASA Jet Propulsion Laboratory, USA

${ }^{3}$ Leibniz-Institut für Oberflächenmodifizierung, Germany

Distinguishing between abiotic and biotic signatures of amino acids and fatty acids on extraterrestrial water worlds is a key prerequisite in the search for life and conditions suitable for its emergence. Cryovolcanically active ocean worlds, such as Saturn's moon Enceladus, can eject water ice grains formed from subsurface water into space. The ice grains provide insights into the oceans' compositions and can be sampled by impact ionization mass spectrometers onboard spacecraft during plume traversals. As well as salts, simple and more complex organics have already been identified in Enceladean ice grains. In previous work we have also shown that biosignatures, such as amino acids, fatty acids and peptides, can similarly be detected in laboratory Laser Induced Liquid Beam Ion Desorption (LILBID) experiments, which are proven to produce mass spectra that are accurate analogues of those generated by impact ionization. These substances could be detected and identified at concentrations equivalent to nanomolar-levels in pure water ice grains.

To determine whether it is possible to discriminate between abiotically and biotically generated compounds in grains arising from an ocean in contact with a rocky core, here we have investigated the LILBID mass spectral appearance and detection limits of amino acids and fatty acids dissolved in an Enceladean ocean-like solution containing other organic and inorganic background components.

In contrast to our previous work, we find that amino acids and fatty acids form characteristic sodiated molecular cations in salty solutions. The detection limits of the organics depend on their $\mathrm{pK}_{\mathrm{a}}$ values and the salinity of the ocean-like solution. Amino acids and fatty acids can be detected at concentrations expected to arise from abiotic and biotic processes in the Enceladean ocean, and the spectral signatures characteristic of abiotically and biotically produced organics can clearly be distinguished from each other. In this work, we devised a methodology to detect in ice grains, and successfully discriminate between, compounds arising from abiotic and biotic processes in extraterrestrial water environments.

Further experimental studies on bacterial DNA and cell material are underway. 